\title{
Liraglutide in the treatment of type 2 diabetes mellitus: clinical utility and patient perspectives
}

This article was published in the following Dove Press journal:

Patient Preference and Adherence

15 March 2010

Number of times this article has been viewed

\author{
Mahamood Edavalath' \\ Jeffrey W Stephens ${ }^{1,2}$ \\ 'Department of Diabetes and \\ Endocrinology, Morriston Hospital, \\ ABM University NHS Trust, Swansea, \\ UK; ' ${ }^{2}$ Diabetes Research Group, \\ Institute of Life Sciences, Swansea \\ University, Swansea, UK
}

\begin{abstract}
Type 2 diabetes mellitus (T2DM) is a progressive disease associated with significant morbidity and mortality. There is good evidence that intensive glycemic control reduces the development and progression of complications in patients with diabetes. In order to achieve glycemic targets, patients often require a combination of oral therapy and/or insulin in addition to lifestyle modification. Unfortunately many currently available therapies for T2DM are associated with weight gain and hypoglycemia resulting in poor compliance and subsequent worsening glycemic control. Glucagon like peptide-1 (GLP-1) is an incretin hormone secreted from the small intestine that lowers fasting and postprandial glucose through multiple mechanisms including glucose-dependent insulin secretion, reduction of glucagon secretion, delaying gastric emptying and increased satiety. Liraglutide is a long acting GLP-1 mimetic that is administered once a day by subcutaneous injection and is now licensed for the treatment of T2DM. Phase 3 clinical trials have demonstrated beneficial effects on glycemic control and weight with liraglutide therapy. Within this article, we provide an overview of pharmacology, efficacy, safety and patient experience on liraglutide in the management of T2DM.
\end{abstract}

Keywords: liraglutide, type 2 diabetes mellitus, glycemic control, GLP-1 analogue, obesity

\section{Introduction}

Type 2 diabetes mellitus (T2DM) is a progressive disease characterized by a variable degree of $\beta$-cell dysfunction, insulin resistance and hyperglycemia. Chronic hyperglycemia plays a major role in the pathogenesis of micro and macrovascular disease, ${ }^{1,2}$ and also potentates $\beta$-cell failure. ${ }^{3}$ Large controlled clinical trials have demonstrated that intensive glycemic control can significantly minimise the development and progression of complications in patients with diabetes. ${ }^{4,5}$ In order to achieve glycemic control, patients with T2DM often require a combination of oral therapy and/or insulin in addition to lifestyle modifications. The United Kingdom Prospective Diabetes Study (UKPDS) ${ }^{6}$ reported that more than $50 \%$ of patients with T2DM will require multiple oral antidiabetic drugs (OAD) within three years after diagnosis. Furthermore, 9 years after diagnosis approximately $70 \%$ of patients required a combination of OAD with or without insulin therapy. This illustrates the complex progressive nature of T2DM which has proved difficult to treat to date.

\section{Liraglutide}

Liraglutide is the second in class of glucagon like peptide-1 (GLP-1) mimetics now available for the treatment of T2DM. The first product licensed was exenatide which 
is a twice-daily delivered functional partly dipeptidyl peptidase-IV (DPP-IV) resistant analogue of human GLP-1 which is now in established use. ${ }^{7}$ GLP-1 mimetics improve glycemic control through multiple mechanisms similar to the endogenous incretin hormone GLP-1. ${ }^{8,9}$ GLP-1 is normally secreted by intestinal L-cells in response to food intake particularly carbohydrate, fat and proteins. It has multiple physiological actions including glucose-dependent insulin secretion and reduced glucagon secretion. In addition, GLP-1 also reduces gastric emptying and increases satiety. ${ }^{10}$ In vivo, GLP-1 is rapidly inactivated by the enzyme DPP-IV resulting in a plasma half-life of 1-2 minutes. To overcome this, DPP-IV resistant analogues of human GLP-1 have been developed for clinical use. Liraglutide is an acylated derivative of GLP-1 currently available for the treatment of T2DM and may be regarded as the only true GLP-1 analogue. Prior to the introduction of incretin based therapies, apart from metformin, all other OAD induce weight gain and an increase in body mass index (BMI) especially in those with pre-existing obesity. In patients with T2DM such an increase in BMI worsens insulin resistance and glycemic control. Furthermore, a raised BMI is an established cause for increased cardiovascular mortality, particularly among individuals with diabetes. ${ }^{11}$ In addition to weight gain, the use of insulin sensitisers such as thiazolidinedione are reported to cause water retention and may exacerbate cardiac failure in some circumstances. Both insulin and insulin secretagogues are known to cause hypoglycemia with associated morbidity and a reduction in quality of life. ${ }^{12}$ These problems of treatment related weight gain, hypoglycemia and fear of hypoglycemia result in poor compliance and deteriorating glycemic control. ${ }^{13,14}$

\section{Pharmacology of liraglutide}

Liraglutide is a long-acting GLP-1 analogue with 97\% sequence homology to human GLP-1. Chemically it is similar to human GLP-1, but with structural modifications including an amino acid substitution of an arginine at position 34 . There is also an addition of a C-16 acyl chain linked to a glutamate spacer at the lysine on position $26 .{ }^{15}$ These structural modifications result in reversible albumin binding, resistance to GLP-1 inactivation by DPP-IV and prolonged duration of action. ${ }^{16}$ Following subcutaneous injection, the absorption of liraglutide is slow with a maximal plasma concentration 9 to 14 hours post delivery with an elimination half life of approximately 13 hours. ${ }^{17}$ Elimination studies have demonstrated that no intact liraglutide and only low levels of metabolites are detected in the urine or feces after administration. ${ }^{17}$ Liraglutide, like other large proteins is degraded in to small peptides, amino acids and fatty acids and eliminated through the liver and kidney. However, no single organ has been identified that plays a major part in elimination and furthermore a proportion of the degraded fragments are recycled into new endogenous proteins and lipids.

\section{Mode of action}

The physiological actions of liraglutide are mediated via specific GLP-1 receptors. Liraglutide shares several glucoregulatory actions with GLP-1 including enhancement of glucose-dependent insulin secretion, inhibition of postprandial glucagon secretion, inhibition of gastric emptying and reduction of food intake. ${ }^{18-21}$ In rodent studies, both GLP-1 and liraglutide promote the maintenance of $\beta$-cell mass in diabetes, presumably by inhibiting both cytokine and free fatty acid induced apoptosis. ${ }^{22}$ Furthermore, clinical studies with liraglutide have been observed to enhance $\beta$-cell function suggesting a potential role in preservation of $\beta$-cell. ${ }^{21}$

\section{Safety and tolerability Beneficial effects of liraglutide Glycemic control}

Treatment with liraglutide is associated with a significant improvement in glycated hemoglobin $\left(\mathrm{HbA}_{1 \mathrm{c}}\right)$, fasting and postprandial glucose. ${ }^{23} \mathrm{Juhl}$ et al, in a double-blind, placebo-controlled, crossover study demonstrated a substantial reduction of fasting and postprandial glycemia following bedtime administration of liraglutide in patients with T2DM. ${ }^{24}$ Within this study the mean fasting plasma glucose was significantly lower with liraglutide compared to placebo $(6.9 \pm 1.0 \mathrm{mmol} / \mathrm{L}$ vs $8.1 \pm 1.0 \mathrm{mmol} / \mathrm{L}, P<0.01)$. Madsbad et al in a randomized, placebo-controlled trial over 12 weeks reported a reduction in both fasting glucose and $\mathrm{HbA}_{1 \mathrm{c}}$ with a once-daily dose of liraglutide. ${ }^{25} \mathrm{In}$ this study the use of $0.75 \mathrm{mg}$ liraglutide resulted in $0.75 \%$ reduction in $\mathrm{HbA}_{1 \mathrm{c}}(P<0.0001)$ and $1.8 \mathrm{mmol} / \mathrm{L}(P=0.0003)$ reduction in fasting glucose compared with placebo. The results of phase III clinical studies - Liraglutide Effects and Action in Diabetes (LEAD) - are available in publication. The LEAD program comprised 6 randomized controlled, double blind studies conducted in more than 40 countries which examined the effect of liraglutide directly against commonly used therapies in T2DM on $\mathrm{HbA}_{1 \mathrm{c}}$ (Table 1). The LEAD-1 study ${ }^{26}$ was a 26 -week, double-dummy, multicenter, multinational 
trial designed to compare the effect of combining liraglutide $(0.6,1.2$ or $1.8 \mathrm{mg} /$ day) or rosiglitazone $4 \mathrm{mg} /$ day or placebo with glimepiride (2-4 mg/day) in patients with T2DM. Liraglutide (1.2 mg or $1.8 \mathrm{mg}$ ) added to glimepiride resulted in a greater reduction in both fasting as well as postprandial plasma glucose from baseline, compared to placebo or rosiglitazone. In addition, liraglutide was associated with a greater reduction in $\mathrm{HbA}_{1 \mathrm{c}}$ from the baseline compared with placebo or rosiglitazone (reduction in $\mathrm{HbA}_{1 \mathrm{c}}$ from baseline $-1.1 \%$ with liraglutide, $+0.2 \%$ with placebo and $-0.4 \%$ with rosiglitazone, $P<0.0001)$. In the LEAD-2 study ${ }^{27}$ the efficacy and safety of liraglutide added to metformin was compared with addition of placebo or glimepiride to metformin. At the end of the 26-week trial period, it was observed that the treatment with liraglutide induced superior glycemic control compared with a placebo group and non inferior glycemic control compared to the glimepiride group.

The LEAD-3 study ${ }^{28}$ was a head to head comparison between liraglutide and glimepiride monotherapy. At 52 weeks, the percentage of patients reaching the American Diabetes Association target $\mathrm{HbA}_{1 \mathrm{c}}$ of less than $7 \%$ were significantly higher in those treated with liraglutide compared to glimepiride monotherapy. Similar results were noted in LEAD-4 trial ${ }^{29}$ when liraglutide was added to metformin and rosiglitazone. Compared to placebo an approximate $0.9 \%$ reduction in $\mathrm{HBA}_{1 \mathrm{c}}$ was observed with $1.2 \mathrm{mg}$ or $1.8 \mathrm{mg}$ of liraglutide compared to placebo $(P<0.0001)$. Furthermore, LEAD-5 study 30 demonstrated a significant improvement in glycemic control by adding liraglutide in patients already receiving metformin and a sulphonylurea compared with placebo and insulin glargine. The reduction in $\mathrm{HBA}_{1 \mathrm{c}}$ was $1.3 \%$ in those receiving $1.8 \mathrm{mg}$ of liraglutide compared to $1.09 \%$ in those receiving glargine insulin $(P=0.0015)$. In LEAD-6 study ${ }^{31}$ the efficacy of liraglutide was compared with the exenatide as add-on therapy to metformin and/or sulphonylurea. Within this study, a greater improvement in glycemic control was noted with once daily liraglutide compared to twice a day exenatide (reduction in mean $\mathrm{HbA}_{1 \mathrm{c}}$ of $1.12 \%$ with liraglutide and $0.7 \%$ with exenatide).

\section{Body weight}

Treatment with liraglutide results in sustained weight loss compared to placebo. This was observed in all of the LEAD studies, except LEAD-1 ${ }^{26-31}$ (Table 1). Of interest, the maximum weight loss of $-3.24 \mathrm{~kg}$ was observed in LEAD-6 which compared the efficacy of liraglutide and exenatide. The observed weight loss with liraglutide appears to be largely independent of nausea. Furthermore, a recent randomized, double-blind, placebocontrolled study conducted in obese individuals without T2DM demonstrated a significant weight reduction associated with liraglutide at 20 weeks. ${ }^{32}$ In this study the mean weight loss observed with liraglutide doses of $1.2 \mathrm{mg}, 1.8 \mathrm{mg}, 2.4 \mathrm{mg}$, and $3.0 \mathrm{mg}$ were $4.8 \mathrm{~kg}, 5.5 \mathrm{~kg}, 6.3 \mathrm{~kg}$, and $7.2 \mathrm{~kg}$ respectively, compared with $2.8 \mathrm{~kg}$ with placebo. Although many studies have demonstrated a significant weight loss with liraglutide, like exenatide it is not currently it is not licensed for use as a weight reducing agent.

\section{$\beta$-cell function}

Liraglutide has been shown to enhance $\beta$-cell mass in animal models. ${ }^{21}$ In addition to animal studies, clinical studies indicate improved $\beta$-cell function as measured by the homeostasis model assessment for $\beta$-cell function (HOMA-B), secretion of C-peptide and proinsulin to insulin ratio. In phase 3 trials, treatment with liraglutide demonstrated significant decrease in the proinsulin to insulin ratio and improvement in HOMA-B from the baseline compared to placebo. ${ }^{26,27,29}$

\section{Cardiovascular system}

A reduction in systolic blood pressure has also been observed with liraglutide as shown in Table $1 .^{27-30}$ The mechanism for this is not well understood but appears to be independent of a reduction in body weight. Like native GLP-1, liraglutide inhibits tumor necrosis factor alpha (TNF) mediated plasminogen activator inhibitor type-1 (PAI-1) activation in human vascular endothelial cells. ${ }^{33}$ These observations suggest that a beneficial effect of liraglutide on endothelial dysfunction associated with premature atherosclerosis observed in T2DM. Evidence from randomized control studies are yet to establish whether such improvement in biomarkers have an impact on long term micro and macrovascular disease.

\section{Adverse effects associated with liraglutide Gastrointestinal}

Like the other GLP-1 receptor agonist exenatide, a major adverse effect of liraglutide therapy is gastrointestinal disturbance with nausea, vomiting, and diarrhea being the commonest reported during clinical trials (Table 2). In early preclinical studies with low doses $(<0.75 \mathrm{mg})$, nausea was reported by $10 / 135$ patients exposed to liraglutide compared to $1 / 29$ patients treated with placebo. ${ }^{25}$ Caution is required with this interpretation at such low doses but 
Table I Changes in $\mathrm{HbA}_{1 \mathrm{c}}$, weight and systolic blood pressure in the LEAD studies

\begin{tabular}{|c|c|c|c|c|}
\hline Study & Number & Change in $\mathrm{HbA}_{\mathrm{Ic}}(\%)$ & Change in body weight (kg) & Change in systolic BP (\%) \\
\hline \multicolumn{5}{|c|}{ LEAD-I26: Liraglutide added on to glimepiride ( 26 weeks duration) } \\
\hline Liraglutide $1.2 \mathrm{mg}$ & 228 & -1.08 & +0.32 & -2.56 \\
\hline Liraglutide $1.8 \mathrm{mg}$ & 228 & -1.13 & -0.23 & -2.81 \\
\hline Rosiglitazone $4 \mathrm{mg}$ & 228 & -0.44 & +2.11 & -0.93 \\
\hline Placebo & 114 & +0.23 & -0.10 & -2.32 \\
\hline \multicolumn{5}{|c|}{ LEAD- $2^{27}$ : Liraglutide added on to metformin ( 26 weeks duration) } \\
\hline Liraglutide $1.2 \mathrm{mg}$ & 228 & -0.97 & -2.58 & -2.81 \\
\hline Liraglutide 1.8 mg & 228 & -1.00 & -2.79 & -2.29 \\
\hline Glimepiride 4 mg & 228 & -0.98 & +0.95 & +0.41 \\
\hline Placebo & 114 & +0.09 & $-|.5|$ & -1.76 \\
\hline \multicolumn{5}{|c|}{ LEAD-328: Liraglutide as monotherapy (52 weeks duration) } \\
\hline Liraglutide $1.2 \mathrm{mg}$ & 234 & -0.84 & -2.05 & -2.12 \\
\hline Liraglutide $1.8 \mathrm{mg}$ & 234 & -1.14 & -2.45 & -3.64 \\
\hline Glimepiride 8 mg & 234 & -0.51 & +1.12 & -0.69 \\
\hline \multicolumn{5}{|c|}{ LEAD- $4^{29}$ : Liraglutide added on to metformin + rosiglitazone ( 26 weeks duration) } \\
\hline Liraglutide $1.2 \mathrm{mg}$ & 178 & -1.48 & -1.02 & -6.71 \\
\hline Liraglutide $1.8 \mathrm{mg}$ & 178 & -1.48 & -2.02 & -5.65 \\
\hline Placebo & 177 & -0.54 & +0.60 & -1.11 \\
\hline \multicolumn{5}{|c|}{ LEAD- $5^{30}$ : Liraglutide added on to metformin + glimepiride ( 26 weeks duration) } \\
\hline Liraglutide $1.8 \mathrm{mg}$ & 207 & -1.33 & -1.81 & -3.97 \\
\hline Glargine & 219 & -1.09 & +1.62 & +0.54 \\
\hline Placebo & 96 & -0.24 & -0.42 & -1.44 \\
\hline \multicolumn{5}{|c|}{ LEAD-6 $6^{31}$ : Liraglutide added on to metformin and/or sulfonylurea ( 26 weeks duration) } \\
\hline Liraglutide $1.8 \mathrm{mg}$ & 233 & -1.12 & -3.24 & -2.51 \\
\hline Exenatide $10 \mu \mathrm{g}$ & 231 & -0.79 & -2.87 & -2.00 \\
\hline
\end{tabular}

Abbreviations: $\mathrm{HbA}_{\mathrm{Ic}}$, hemoglobin $\mathrm{A}_{\mathrm{Ic}}$; $\mathrm{BP}$, blood pressure.

nevertheless, in this study, approximately two-thirds of reported gastrointestinal events resolved within 1 to 3 days of continuing therapy and no patients withdrew from the treatment due to gastrointestinal events. More clinically relevant doses prescribed in the phase 3 clinical trials have reported the incidence of nausea with liraglutide to range from $10 \%$ to $40 \%$ compared to $2 \%$ to $9 \%$ with the placebo group. ${ }^{26-29}$ In these trials nausea occurred early during treatment and with continued therapy the frequency and severity decreased in majority of patients after the initial 4 weeks of therapy. Similarly, diarrhea, vomiting, dyspepsia and constipation were often mild and noted to diminish within few days or weeks on continued therapy. The percentage of patients withdrawn because of gastrointestinal side effects like nausea, vomiting and diarrhea was low, ranging from $2 \%$ to $5 \% .{ }^{26-28}$ However, in LEAD-4 trial, ${ }^{29}$ the rate of withdrawal was high at $11 \%$ with the higher dose of liraglutide $(1.8 \mathrm{mg} /$ day $)$. Since the gastrointestinal adverse effects are transient and dose depended liraglutide should be titrated from $0.6 \mathrm{mg}$ at weekly increments.

\section{Pancreatitis}

Three cases of pancreatitis ( 1 patient in LEAD-1 and 2 patients in LEAD-3) have been reported during clinical trials with liraglutide. Compared to general population, patients with T2DM have a three fold risk of acute pancreatitis. ${ }^{34}$ Therefore, it is difficult to establish whether these reported cases are due to liraglutide therapy or to pre-existing increased risk. In view of confounding factors a causal relationship between liraglutide and pancreatitis may be difficult to establish however, a weak association cannot be excluded based on available clinical data. ${ }^{27,28} \mathrm{~A}$ similar view has been expressed for exenatide. ${ }^{35}$

\section{Hypoglycemia}

Minor hypoglycemic episodes were observed with liraglutide therapy during phase 3 clinical trials (Table 2). The frequency 
Table 2 Nausea and hypoglycemia as common adverse effects in the LEAD studies

\begin{tabular}{|c|c|c|c|}
\hline$\overline{\text { Study }}$ & Nausea (\%) & aHypoglycemia major (\%) & 'bypoglycemia minor (\%) \\
\hline \multicolumn{4}{|l|}{ LEAD- $\left.\right|^{26}$} \\
\hline Liraglutide $1.2 \mathrm{mg}$ & 10.5 & 0 & 9.2 \\
\hline Liraglutide $1.8 \mathrm{mg}$ & 6.8 & 0.4 & 8.1 \\
\hline Rosiglitazone $4 \mathrm{mg}$ & 1.7 & 0 & 4.3 \\
\hline Placebo & 2.5 & 0 & 2.6 \\
\hline \multicolumn{4}{|l|}{ LEAD-2 ${ }^{27}$} \\
\hline Liraglutide $1.2 \mathrm{mg}$ & 16.2 & 0 & 3.3 \\
\hline Liraglutide $1.8 \mathrm{mg}$ & 17.7 & 0 & 6.6 \\
\hline Glimepiride 4 mg & 3.3 & 0 & 26.9 \\
\hline Placebo & 3.3 & 0 & 5.8 \\
\hline \multicolumn{4}{|l|}{ LEAD- $3^{28}$} \\
\hline Liraglutide $1.2 \mathrm{mg}$ & 27.5 & 0 & 11.2 \\
\hline Liraglutide $1.8 \mathrm{mg}$ & 29.3 & 0 & 17.7 \\
\hline Glimepiride 8 mg & 8.5 & 0 & 24.2 \\
\hline \multicolumn{4}{|l|}{ LEAD- $4^{29}$} \\
\hline Liraglutide $1.2 \mathrm{mg}$ & 30.5 & 0 & 9.0 \\
\hline Liraglutide $1.8 \mathrm{mg}$ & 40.4 & 0 & 7.9 \\
\hline Placebo & 8.6 & 0 & 5.1 \\
\hline \multicolumn{4}{|l|}{ LEAD- $5^{30}$} \\
\hline Liraglutide $1.8 \mathrm{mg}$ & 13.9 & 2.2 & 27.4 \\
\hline Glargine & 1.3 & 0 & 28.9 \\
\hline Placebo & 3.5 & 0 & 16.7 \\
\hline \multicolumn{4}{|l|}{ LEAD- $6^{3 !}$} \\
\hline Liraglutide $1.8 \mathrm{mg}$ & 26.0 & 0 & 26.0 \\
\hline Exenatide $10 \mu \mathrm{g}$ & 28.0 & 0.9 & 34.0 \\
\hline
\end{tabular}

aHypoglycemic episodes requiring third-party assistance.

bSelf treated hypoglycemic episodes.

of hypoglycemia varied depending on the oral hypoglycemic agents co-administered. This is in line with studies relating to exenatide. ${ }^{36}$ In a recent randomized controlled trial, it was reported that the incidence of minor hypoglycemia with liraglutide was comparable (3\%) to placebo and $17 \%$ lower than with sulphonylurea. ${ }^{27}$ Another phase 3 trial reported minor hypoglycemia at a rate less than $<0.5$ episodes/patient year for liraglutide compared to 1.96 episodes/patient year with glimepiride. ${ }^{28}$ In a comparative study of liraglutide against insulin glargine when added to metformin and glimepiride, the incidence of minor hypoglycemia was similar (27\% with liraglutide and 29\% with glargine).$^{30}$ Within this study, 2.2\% patients in the liraglutide treated arm had major hypoglycemia (none of these was nocturnal hypoglycemia) compared to none in the glargine group. Similarly, studies with exenatide have described an increased risk of hypoglycemia when co-administered with a sulphonylurea. ${ }^{37,38}$ Of interest, a study in mice examining
GLP-1 in relation to tolbutamide, observed that uncoupling of GLP-1 from its glucose dependence was observed with tolbutamide. ${ }^{39}$ It is possible that such a mechanism may play a role in the increased hypoglycemia observed in diabetic patients receiving combination therapy of sulphonylurea and a GLP-1 agonist. Therefore, both patients and health carers should exercise increased vigilance for this potential adverse effect particularly when using combination of liraglutide with sulphonylurea.

\section{Immunogenicity}

Treatment with liraglutide may elicit an immune response leading to anti-liraglutide antibody production. During phase 3 clinical trials anti-liraglutide antibodies have been detected in $4 \%$ to $13 \%$ of liraglutide recipients; ${ }^{26,28,29}$ however, these antibodies did not appears to alter the efficacy of liraglutide among the trial subjects. Moreover, compared to exenatide $(41 \%-49 \%)$ the incidence of antibody formation is 
significantly lower with liraglutide treatment which is thought to be due to the high degree of homology of liraglutide to human GLP-1. ${ }^{36}$

\section{Lipohypertrophy}

Lipohypertrophy is a common problem associated with long-term insulin therapy. ${ }^{40,41}$ There are no studies available yet to confirm such skin changes are possible with liraglutide therapy. In a previous clinical trial, the subcutaneous use of liraglutide is noted to cause injection site bruising however, the frequency was no different compared to placebo injection (5.7\% vs $5.9 \%$ of participants). ${ }^{42}$ In another study, ${ }^{25}$ mild injection site urticarial reaction was noted in 1 of the 135 participants treated with liraglutide.

\section{Children, pregnancy and lactation}

The use of liraglutide has not been studied in pediatrics and its use is not recommended for those aged below 18 years. There are no controlled trials examining the effects of liraglutide on fertility or its teratogenic potential in pregnant women. Fertility studies in animals did not show a direct harmful effect on fertility; however, maternal administration of liraglutide at mid-gestation caused minor and reversible skeletal changes in fetal rats and rabbits. The safety of liraglutide during lactation has not been established. Current recommendation by manufacturers is not to use liraglutide during pregnancy and breast feeding.

\section{Patient reported outcomes}

Much of the available information on patient outcomes reported to date are in abstract form. Within LEAD-2, the Diabetes Treatment Satisfaction Questionnaire (DTSQ) and Impact of Weight on Quality Of Life (IWQOL-Lite) were examined in relation to liraglutide as add-on therapy to metformin compared to placebo or glimepiride. ${ }^{43}$ Liraglutide was associated with a significant reduction in perceived hypoglycemia and hyperglycemia. Furthermore, there was a significant reduction in the public stress domain (ie, distress about weight when out in public) in those who received liraglutide. Within the LEAD-6 study, patient reported outcomes were recorded in 379 patients using the Diabetes Treatment Satisfaction Questionnaire score (DTSQs). ${ }^{44}$ It was observed that the highest treatment satisfaction was with liraglutide compared to exenatide $(P<0.0001)$. Of interest within the questionnaire, liraglutide was superior to exenatide in respect to 'current treatment', 'convenience', 'flexibility', 'recommend' and 'continue'. The proportion of 'satisfied' patients (DTSQ-change >6) was 94\% with liraglutide compared to $86 \%$ with exenatide $(P=0.0176)$. Patients also perceived significantly less hypoglycemia and hyperglycemia. Of note, in the extension phase of the trial the DTSQs remained stable in those who continued on liraglutide and improved significantly in those who switched from exenatide to liraglutide.

\section{Conclusion}

It is evident from the phase 3 clinical trials that treatment with liraglutide is associated with significant improvement in fasting and postprandial glucose and $\mathrm{HbA}_{1 \mathrm{c}}$. In addition, liraglutide is shown to induce satiety and promote weight loss. Hence for patients not controlled by 'traditional' OADs, liraglutide is well designed to target many of the phenotypic features associated with T2DM. The relative weight loss compared to typical weight gain with insulin, sulphonylurea and thiazolidinedione therapy is clearly a benefit in T2DM. Liraglutide is well tolerated and the most common adverse effect like gastrointestinal disturbances are transient and dose dependent. Therefore, it is recommended that liraglutide should be titrated from $0.6 \mathrm{mg}$ at weekly increments. It would appear from the available literature to date (summarized in Table 1) that the overall reduction in $\mathrm{HBA}_{1 \mathrm{c}}$ is similar between the $1.2 \mathrm{mg}$ and $1.8 \mathrm{mg}$ dose, but there is clearly a price increment between these doses. Of interest the withdrawal rate from trials was also higher with the higher dose. This may explain why certain health boards and primary care trusts currently recommend use at a dose up to the $1.2 \mathrm{mg}$. Although the incidence of hypoglycemia is low with liraglutide, caution is required with co-existing sulphonylurea use as hypoglycemia may be more frequent. Compared to the recommended twice daily injections of exenatide, liraglutide with its once daily use may provide an additional advantage within this class of agents and might enhance patient acceptability and compliance.

\section{Disclosures}

The authors disclose no conflicts of interest.

\section{References}

1. Creager MA, Luscher TF, Cosentino F, Beckman JA. Diabetes and vascular disease: pathophysiology, clinical consequences, and medical therapy: Part I. Circulation. 2003;108(12):1527-1532.

2. Sheetz MJ, King GL. Molecular understanding of hyperglycemia's adverse effects for diabetic complications. JAMA. 2002;288(20): 2579-2588. 
3. Grill V, Bjorklund A. Overstimulation and beta-cell function. Diabetes. 2001;50 Suppl 1:S122-S124.

4. The effect of intensive treatment of diabetes on the development and progression of long-term complications in insulin-dependent diabetes mellitus. The Diabetes Control and Complications Trial Research Group. N Engl J Med. 1993;329(14):977-986.

5. Intensive blood-glucose control with sulphonylureas or insulin compared with conventional treatment and risk of complications in patients with type 2 diabetes (UKPDS 33). UK Prospective Diabetes Study (UKPDS) Group. Lancet. 1998;352(9131):837-853.

6. Turner RC, Cull CA, Frighi V, Holman RR. Glycemic control with diet, sulfonylurea, metformin, or insulin in patients with type 2 diabetes mellitus: progressive requirement for multiple therapies (UKPDS 49). UK Prospective Diabetes Study (UKPDS) Group. JAMA. 1999;281(21):2005-2012.

7. Keating GM. Exenatide. Drugs. 2005;65(12):1681-1692; discussion 1693-1685.

8. Drucker DJ. Enhancing incretin action for the treatment of type 2 diabetes. Diabetes Care. 2003;26(10):2929-2940

9. Joy SV, Rodgers PT, Scates AC. Incretin mimetics as emerging treatments for type 2 diabetes. Ann Pharmacother. 2005;39(1):110-118.

10. Holst JJ. The physiology and pharmacology of incretins in type 2 diabetes mellitus. Diabetes Obes Metab. 2008;10(Suppl 3):14-21.

11. Whitlock G, Lewington S, Sherliker P, et al. Body-mass index and cause-specific mortality in 900,000 adults: collaborative analyses of 57 prospective studies. Lancet. 28 2009;373(9669):1083-1096.

12. Amiel SA, Dixon T, Mann R, Jameson K. Hypoglycaemia in Type 2 diabetes. Diabet Med. 2008;25(3):245-254.

13. Davis S, Alonso MD. Hypoglycemia as a barrier to glycemic control. J Diabetes Complications. 2004;18(1):60-68.

14. Green J, Feinglos M. Update on type 2 diabetes mellitus: understanding changes in the diabetes treatment paradigm. Int J Clin Pract Suppl. 2007;154:3-11.

15. Knudsen LB, Nielsen PF, Huusfeldt PO, et al. Potent derivatives of glucagon-like peptide-1 with pharmacokinetic properties suitable for once daily administration. $J$ Med Chem. 2000;43(9):1664-1669.

16. Gonzalez C, Beruto V, Keller G, Santoro S, Di Girolamo G. Investigational treatments for Type 2 diabetes mellitus: exenatide and liraglutide. Expert Opin Investig Drugs. 2006;15(8):887-895.

17. Agerso H, Jensen LB, Elbrond B, Rolan P, Zdravkovic M. The pharmacokinetics, pharmacodynamics, safety and tolerability of NN2211, a new long-acting GLP-1 derivative, in healthy men. Diabetologia. 2002;45(2):195-202.

18. Kreymann B, Williams G, Ghatei MA, Bloom SR. Glucagonlike peptide-1 7-36: a physiological incretin in man. Lancet. 1987;2(8571):1300-1304.

19. Wettergren A, Schjoldager B, Mortensen PE, Myhre J, Christiansen J, Holst JJ. Truncated GLP-1 (proglucagon 78-107-amide) inhibits gastric and pancreatic functions in man. Dig Dis Sci. 1993;38(4): 665-673.

20. Naslund E, Gutniak M, Skogar S, Rossner S, Hellstrom PM. Glucagon-like peptide 1 increases the period of postprandial satiety and slows gastric emptying in obese men. Am J Clin Nutr. 1998;68(3):525-530.

21. Bregenholt S, Moldrup A, Blume N, et al. The long-acting glucagonlike peptide-1 analogue, liraglutide, inhibits beta-cell apoptosis in vitro. Biochem Biophys Res Commun. 2005;330(2):577-584.

22. Mari A, Degn K, Brock B, Rungby J, Ferrannini E, Schmitz O. Effects of the long-acting human glucagon-like peptide-1 analog liraglutide on beta-cell function in normal living conditions. Diabetes Care. 2007;30(8):2032-2033.

23. Seino Y, Rasmussen MF, Zdravkovic M, Kaku K. Dose-dependent improvement in glycemia with once-daily liraglutide without hypoglycemia or weight gain: A double-blind, randomized, controlled trial in Japanese patients with type 2 diabetes. Diabetes Res Clin Pract. 2008;81(2):161-168.
24. Juhl CB, Hollingdal M, Sturis J, et al. Bedtime administration of NN2211, a long-acting GLP-1 derivative, substantially reduces fasting and postprandial glycemia in type 2 diabetes. Diabetes. 2002;51(2):424-429.

25. Madsbad S, Schmitz O, Ranstam J, Jakobsen G, Matthews DR. Improved glycemic control with no weight increase in patients with type 2 diabetes after once-daily treatment with the long-acting glucagonlike peptide 1 analog liraglutide (NN2211): a 12-week, double-blind, randomized, controlled trial. Diabetes Care. 2004;27(6):1335-1342.

26. Marre M, Shaw J, Brandle M, et al. Liraglutide, a once-daily human GLP-1 analogue, added to a sulphonylurea over 26 weeks produces greater improvements in glycaemic and weight control compared with adding rosiglitazone or placebo in subjects with Type 2 diabetes (LEAD-1 SU). Diabet Med. 2009;26(3):268-278.

27. Nauck M, Frid A, Hermansen K, et al. Efficacy and safety comparison of liraglutide, glimepiride, and placebo, all in combination with metformin, in type 2 diabetes: the LEAD (liraglutide effect and action in diabetes)-2 study. Diabetes Care. 2009;32(1):84-90.

28. Garber A, Henry R, Ratner R, et al. Liraglutide versus glimepiride monotherapy for type 2 diabetes (LEAD-3 Mono): a randomised, 52-week, phase III, double-blind, parallel-treatment trial. Lancet. 2009;373(9662):473-481.

29. Zinman B, Gerich J, Buse JB, et al. Efficacy and safety of the human glucagon-like peptide-1 analog liraglutide in combination with metformin and thiazolidinedione in patients with type 2 diabetes (LEAD-4 Met+TZD). Diabetes Care. 2009;32(7):1224-1230.

30. Russell-Jones D, Vaag A, Schmitz O, et al. Liraglutide vs insulin glargine and placebo in combination with metformin and sulfonylurea therapy in type 2 diabetes mellitus (LEAD- 5 met+SU): a randomised controlled trial. Diabetologia. 2009;52(10):2046-2055.

31. Buse JB, Rosenstock J, Sesti G, et al. Liraglutide once a day versus exenatide twice a day for type 2 diabetes: a 26-week randomised, parallel-group, multinational, open-label trial (LEAD-6). Lancet. 2009;374(9683):39-47.

32. Astrup A, Rossner S, Van Gaal L, et al. Effects of liraglutide in the treatment of obesity: a randomised, double-blind, placebo-controlled study. Lancet. 2009;374(9701):1606-1616.

33. Liu H, Dear AE, Knudsen LB, Simpson RW. A long-acting glucagonlike peptide-1 analogue attenuates induction of plasminogen activator inhibitor type-1 and vascular adhesion molecules. $J$ Endocrinol. 2009;201(1):59-66.

34. Noel RA, Braun DK, Patterson RE, Bloomgren GL. Increased risk of acute pancreatitis and biliary disease observed in patients with type 2 diabetes: a retrospective cohort study. Diabetes Care. 2009;32(5): 834-838.

35. Bain SC, Stephens JW. Exenatide and pancreatitis: an update. Expert Opin Drug Saf. 2008;7(6):643-644.

36. Stephens JW, Bain SC. Safety and adverse effects associated with GLP-1 analogues. Expert Opin Drug Saf. 2007;6(4):417-422.

37. Kendall DM, Riddle MC, Rosenstock J, et al. Effects of exenatide (exendin-4) on glycemic control over 30 weeks in patients with type 2 diabetes treated with metformin and a sulfonylurea. Diabetes Care. 2005;28(5):1083-1091.

38. Heine RJ, Van Gaal LF, Johns D, Mihm MJ, Widel MH, Brodows RG. Exenatide versus insulin glargine in patients with suboptimally controlled type 2 diabetes: a randomized trial. Ann Intern Med. 2005;143(8): $559-569$.

39. de Heer J, Holst JJ. Sulfonylurea compounds uncouple the glucose dependence of the insulinotropic effect of glucagon-like peptide 1. Diabetes. 2007;56(2):438-443.

40. Hauner H, Stockamp B, Haastert B. Prevalence of lipohypertrophy in insulin-treated diabetic patients and predisposing factors. Exp Clin Endocrinol Diabetes. 1996;104(2):106-110.

41. Ruvalcaba RH, Kletter GB. Abdominal lipohypertrophy caused by injections of growth hormone: a case report. Pediatrics. 1998; 102(2 Pt 1):408-410. 
42. Feinglos MN, Saad MF, Pi-Sunyer FX, An B, Santiago O. Effects of liraglutide (NN2211), a long-acting GLP-1 analogue, on glycaemic control and bodyweight in subjects with Type 2 diabetes. Diabet Med. 2005;22(8):1016-1023.

43. Frid A NM, Hermansen K, Kolotkin RL, Hammer M, Zdravkovic M, Matthews D. Evaluation of patient reported outcomes in subjects with type 2 diabetes treated with the once-daily human GLP-1 analog liraglutide or glimepiride, both as add-on to metformin. American Diabetes Association's 68th Scientific Sessions; June 2008. Abstract 2080-PO.
44. Schmidt WE CJ, Hammer M, Zychma M, Buse JB. Patient-reported outcoes are superior in patients with type 2 diabetes treated with liraglutide as compared to exenatide, when added to metformin, sulfonylurea or both (LEAD-6). American Diabetes Association's 69th Scientific Sessions; June 2009. Abstract P-1880.

\section{Publish your work in this journal}

Patient Preference and Adherence is an international, peer-reviewed, open access journal that focusing on the growing importance of patient preference and adherence throughout the therapeutic continuum. Patient satisfaction, acceptability, quality of life, compliance, persistence and their role in developing new therapeutic modalities and compounds to optimize clinical outcomes for existing disease states are major areas of interest. This journal has been accepted for indexing on PubMed Central. The manuscript management system is completely online and includes a very quick and fair peer-review system. Visit http://www.dovepress.com/ testimonials.php to read real quotes from published authors.

Submit your manuscript here: http://www.dovepress.com/patient-preference-and-adherence-journal 